\title{
ЧИСЛЕННОЕ ИССЛЕДОВАНИЕ ВЯЗКОСТНО-ИНЕРЦИОННОГО ЛАМИНАРНОГО ЗАКРУЧЕННОГО ТЕЧЕНИЯ В КРУГЛОЙ ТРУБЕ С ЭКСЦЕНТРИЧНЫМ КРУГЛЫМ ЯДРОМ
}

\author{
Харламов Сергей Николаевич1', \\ kharsn@mail.ru \\ Джангхорбани Мехран ${ }^{1}$,
mehran.janghorbani@gmail.com \\ 1 Национальный исследовательский Томский политехнический университет, \\ Россия, 634050, г. Томск, пр. Ленина, 30.
}

\begin{abstract}
Актуальность исследования определяется необходимостью понимания особенностей гидродинамики и тепломассообмена в реологически сложных однородных и неоднородных средах при течении в кольцевых областях. Это важно для выработки рекомендаций по управлению бурением, повышения надежности функкционирования специального оборудования при высоких динамических и тепловых нагрузках при вращении трубы, заполнения межтрубного пространства шламом, а также установления контроля за изменением состава, структуры, давления, скорости и реофизических свойств (с псевдопластическими, тиксотропными и вязкоэластичными эффректами) бурового раствора на горизонтальных участках скважин.

Цель: исследовать гидродинамику вязкого потока в кольцевых трубах с эксцентрично расположенным круглым ядром, полых каналах в условиях прямоточного и закрученного (способом подвижной внутренней стенки/локально на входе) течений; внести понимание эффректов, сопровождающих операции бурения на наклонных и горизонтальных участках скважин с эксцентричными бурильными трубами; установить особенности изменений динамической структуры потока в зонах преимущественного движения вязких сред за счет инерционных сил, а также в моменты торможения потока при загромождении сечения продуктами выработки; выдать рекомендации о благоприятном воздействии инерционных сил на вязкость промывочных жидкостей для поддержания эфффективного бурения нефтяных скважин.

Методы: инженерный анализ моделей реальности процессов транспорта реологически сложных вязких сплошных сред во внутренних системах (трубах, каналах) и их описание методами физико-математического и численного моделирования 8 форме систем диффференциальных и алгебраических уравнений, решение которых в важнейших аспектах технологических процессов бурения согласуется с характеристиками элементов специального оборудования.

Результаты. Исследованы внутренние течения вязких сред со специфической реологией [ньютоновские и неньютоновские жидкости (типа Гершеля-Балкли)] в геометрических конфигурациях, характерных для эксцентричных бурильных труб с эфрфектами от изменений их пространственной ориентации, расхода, интенсивности вращения входящего потока/стенки ядра, а также реосизических свойств $(т,, \kappa, n)$. Параметрический анализ динамических эфффектов выполнен для диапазона изменений критериев: Россби Ro=0...5, Рейнольдса $R e=10^{2} \ldots 1^{3}$, Бингама $B i=5 \ldots 15$, эксцентриситета $\Delta=0,1 \ldots 0,9$. Оценены и обобщены эфффекты от механизмов конвективно-дифффузионного взаимодействия процесса переноса импульса в трубах/каналах при сложном движении потока и его контактах со стенками. Расчеты показывают, что любые осложнения течения капельных сред обусловлены изменением полей давления, скорости, внешних и внутренних сил (вследствие реологии). Проанализированы особенности возникновения рециркуляционных зон в закрученном потоке, затухания по длине трубы тангенциальной компоненты скорости. Отмечается, что с ростом эксиентриситета ядра усиливается неоднородность потока, асимметрия распределения осевой компоненты вектора скорости и устанавливаются условия к блокированию течения в нижней части межтрубного пространства. Установлено, что наличие препятствий движению потока в кольцевых областях, например, в виде частии шлама при бурении, способно интенсифицировать асимметричность процессов переноса, особенно при высоких числах Re, Bi. Этого можно избежать в режимах течения с вращением бурильной трубы методом подвижной стенки/орбитального движения. В заключение приведены рекомендации по моделированию, расчету течений вязких сред, сопровождающих бурение, очистку горизонтальных скважин.
\end{abstract}

\section{Ключевые слова:}

Скважина, бурение, вращение, моделирование, гидродинамика, реология, кольцевые потоки, транспорт, очистка.

\section{Введение}

Во многих динамических процессах, оказывающих существенное влияние на функционирование промышленного оборудования, участвуют потоки вязких сред, представляющих собой системы/смеси со специфической реологией, структурой и составом твердых частиц неодинакового размера и формы. Течение таких сред по пространству рабочей области (в каналах, трубах, щелях, кольцевых участках/элементах рабочих узлов с эксцентрично расположенным круглым ядром) осложнено процессами осаждения/загромождения проходного сечения из-за высокой зависимости скорости потока от характера изменений внутренних и внешних сил, возмущений, генерируемых на грани- цах. В нефтегазовой и химико-технологической промышленности актуальны проблемы повышения эффективности оборудования, обеспечивающего очистку скважин от бурового шлама [1-3]. Прогноз режимов гидродинамической очистки скважин от шлама в кольцевых областях межтрубного пространства ставит задачи определения сути гидродинамических эффектов, формирующих устойчивые режимы течения смесей со взвешенными твердыми частицами посредством наложения на прямоточное течение эффектов вращения внутренней трубы. В таких условиях характер движения смеси будет существенно зависеть от интенсивности закрутки и эксцентриситета круглого ядра, силы тяжести, особенностей конвективно- 
диффузионного взаимодействия частиц смеси как между собой, так и со стенками межтрубного пространства. Процессы гравитационного и центробежного осаждения мелких частиц в жидкости, условия захвата и уноса их вязким потоком требуют изучения особенностей перестройки по пространству однородного и неоднородного закрученного потока, определения их закономерностей. В таких условиях вполне естественным представляется установление деталей движения первоначально гомогенной химически инертной среды (несжимаемой жидкости) в коаксиальном межтрубном пространстве постоянного поперечного сечения и последующее обобщение данных на более общую геометрическую и физическую конфигурацию, характерную для специальных задач гидродинамической очистки скважин от шлама. Видно [1-4], что при организации эффективного и оптимального управления операциями нефтегазового бурения роль эффектов от гидродинамики и теплообмена в вязкостно-инерционно-гравитационном течении жидкостей в элементах специального оборудования значительна и их изучение актуально. Однако в отношении процессов бурения все еще отсутствуют полные и достоверные сведения о деталях влияния закрутки и реологии среды на транспортировку бурового раствора по стволу скважины. Результаты исследования закрученных внутренних вязкостноинерционных потоков (локально на входе, вращающейся стенки вокруг своей продольной оси) показывают (например, [5-9]), что в таких потоках формируются области со специфическим рециркуляционным характером изменения поля скорости, вызванным особенностями изменения поля давления. Эти процессы существенно влияют на эффекты отрыва потока, экстенсификации процессов переноса, формируют пространственные размеры застойных областей, способствуют образованию отложений частиц на стенках и служат источником для установления механизмов седиментациии, сальтации твердых частиц в гетерогенной смеси.

Таким образом, иель работы заключалась в: численном исследовании гидродинамики вязкого потока в трубе с эксцентрично расположенным круглым ядром в условиях прямоточного и закрученного (способом подвижной внутренней стенки) течений; выяснении роли установления особенностей и закономерностей механизмов, определяющих перестройку поля скорости по кольцевому пространству; выдаче рекомендащии по прогнозу изменений локальных и интегральных параметров течения, обеспечивающих эффективность транспорта частиц жидкости и оказывающих благоприятное воздействие на реологические свойства промывочных жидкостей при бурении нефтяных скважин.

\section{Физическая и математическая постановка задачи}

В общем случае вязкостно-инерционно-гравитационного ламинарного движения несжимаемой жидкости по кольцевой области межтрубного пространства с эксцентричным круглым ядром допускается, что течение ньютоновской/неньютоновской жидкости в условиях отсутствия силы тяжести и эксцентриси- тета вращательно-симметрично. Гидродинамика и теплообмен жидкости со стенками канала осложнены влиянием переменности теплофизических свойств, действием внешних сил (гравитационных и центробежных), особенностями реологической связи напряжений со скоростями деформаций, нестационарными эффектами, формируемыми характером движения круглого ядра и интенсивностью его вращения как вокруг собственной продольной оси (с центром $O_{1}$ ), так и ее неустановившегося/стационарного орбитального движения относительно продольной оси кольцевого канала (с центом $O_{2}$ ). Исследование особенностей течения проводится в режимах гидродинамики и теплообмена, характерных для технологических операций, физических процессов, сопровождающих функционирование бурового оборудования, движений бурового раствора на горизонтальных участках скважин, с целью организации эффективных операций транспорта шлама и его уноса из низкорейнольдсовых и высоковязких пристеночных областей.

Детали движения и области расчета жидкости

Геометрическая конфигурация области гомогенного/гетерогенного течения ньютоновской (вода)/неньютоновской жидкости (Гершеля-Балкли, Оствальда-де Вале) в аксиальном направлении типична для кольцевых каналов и включает параметры: длины $(L)$ поверхности; радиусов внешней $\left(R_{2}\right)$ и внутренней $\left(R_{1}=\varkappa R_{2}\right)$ круглых цилиндрических труб; кольцевого зазора $\left(a=R_{2}-R_{1}\right)$; расстояния $\left(b=O_{2}-O_{1}\right)$ между центрами внешней трубы $\left(O_{2}\right)$ и круглого ядра $\left(O_{1}\right)$; эксцентриситета ядра $-e=\frac{2 b}{D-d}$. Особенности изменений структуры внутреннего потока и характера движения вязкой среды/буровой смеси в скважине при вязкостно-инерционно-гравитационном течении, осложненном вращением ядра, представлены в [1-9], и дополнительно предполагается, что имеется $a$ - минимальный зазор между внешней трубой и ядром, при котором возможен транспорт вязкой среды по всему сечению кольцевого пространства; $y, z$ - соответственно радиальная и азимутальная независимые переменные декартовой системы координат; $\varphi$ - переменная цилиндрической системы/угол, характеризующая процессы в радиальной плоскости межтрубного пространства; $\varphi_{\text {я }}$ переменная изменения центра ядра при орбитальном движении; $r_{0}$ - радиус кольцевой зоны интенсивного течения. Общий анализ имеющихся библиографических данных по рассматриваемой проблеме (например, [1-15]) позволяет отметить, что транспорт вязкой жидкости с ньютоновской реологией по межтрубному пространству, свободному от примесей, происходит без существенных особенностей, за исключением специфики изменения поля скорости смеси в низкорейнольдсовой и эксцентричной (нижней) части кольцевой зоны. При динамике неньютоновских сред следует учитывать, что превышение максимального касательного напряжения $\left(\tau_{0}\right)$ и предельного статического напряжения сдвига (деформаций, $\theta$ ) влечет интенсификацию течения в областях, отвечающих части сечения кольцевого про- 
странства, которую можно ограничить размерами дуг большой и малой окружностей с радиусами внешней трубы $\left(R_{2}\right)$ и круглого ядра $\left(R_{1}\right)$, связанных между собой пространством с окружностями радиуса $r_{0}$. Эта область живого сечения определяется относительно большим спектром изменений скоростей течения, а также скоростей деформаций, которые способны генерировать высокую циркуляцию в тиксотропной жидкости. В случае орбитального движения ядра относительно центра $O_{2}$ продольной оси внешней трубы его положение следует связать с изменением центра ядра $O_{1}$ по окружности радиуса $b$ в зависимости от угла $\varphi_{я}$. Заметим, что в режимах течения с закруткой стенки ядра его взаимодействия с тиксотропной жидкостью в пристеночной области будут формировать дополнительные напряжения, интенсифицирующие вязкостно-инерционно-гравитационные механизмы переноса импульса, тепла, массы. В таких условиях в физической и математической постановках задачи необходимо учитывать дополнительные нестационарные эффекты при течении капельной среды и связывать их с неустановившемся движением центра $O_{1}$ по окружности (с отклонением $b=O_{2}-O_{1}$ и изменением текущего угла $\left.\varphi_{я}=\varphi_{я}(t)\right)$.

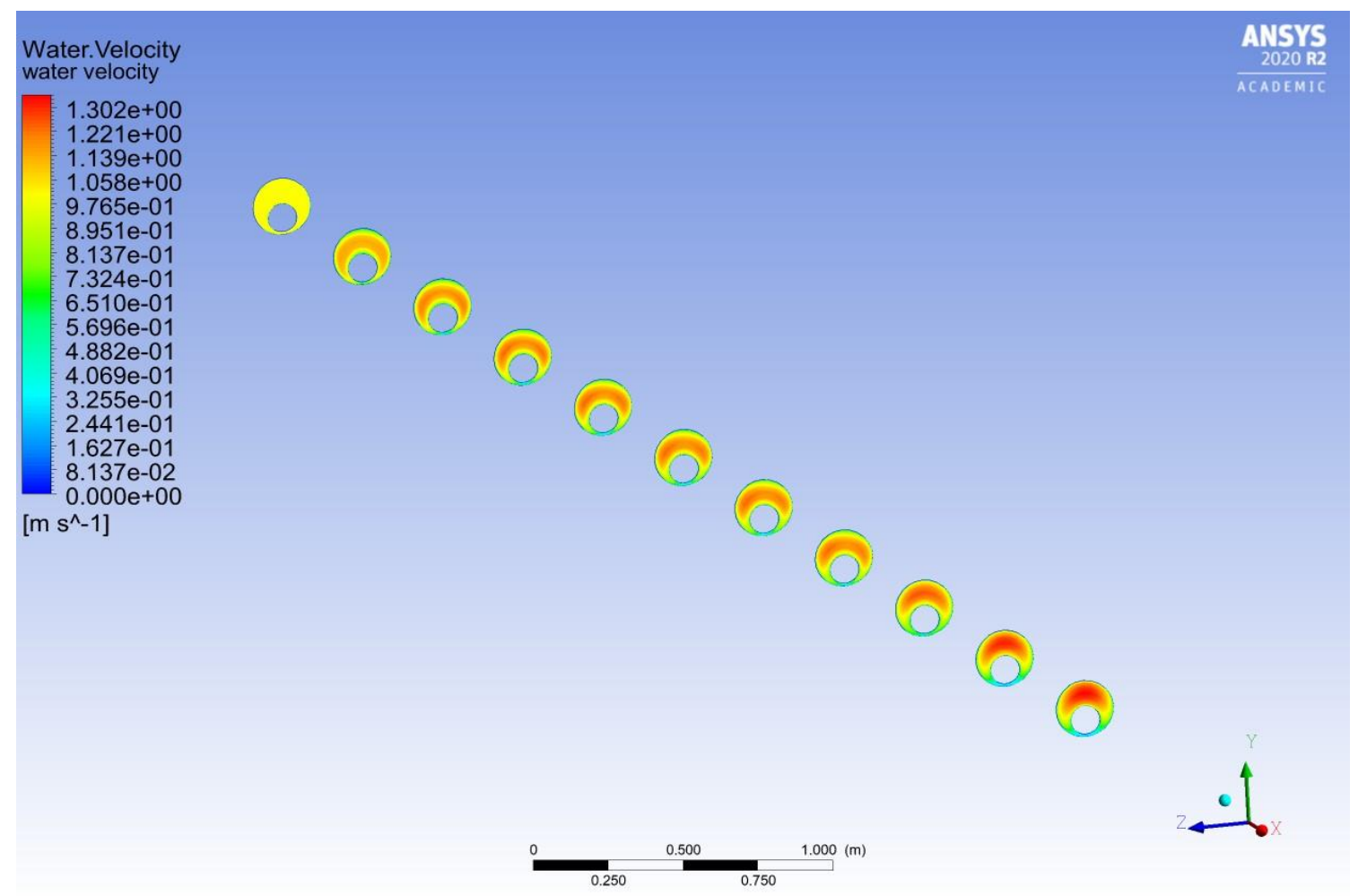

Puс. 1. Характерная картина изменения поля скорости при прямоточном вязкостно-инериионном течении капельной ньютоновской жидкости (вода) в эксиентричной трубе без внешних сил, при ударном профиле на входе

Fig. 1. Characteristic picture of the change in velocity field in the direct viscous-inertial flow of the Newtonian liquid (water) in the eccentric tube at the «shock» entrance profile and without external forces

Детальное исследование процессов бурения скважин с фиксированным эксцентриситетом бурильной трубы, выполненное, например, в [12], также указывает, что начало движения в реологически сложной среде в эксцентричной области происходит с учетом значений поля давления, имеющего порядок $O\left(\theta / R_{h}\right)$, где $R_{h}$ - величина гидравлического радиуса радиального сечения трубы, включающего выраженную циркуляцию потока. Причем его максимальное значение определяется частью площади кольцевой зоны, в которой происходит циркуляция, и она зависит от эксцентричности круглого ядра. Следует ожидать, что если процесс происходит при постоянных параметрах $\theta$ и $R_{h \max }$, то будем иметь восстановление циркуляции при минимальном градиенте давления. Также из [13] следует важное для моделирования положение, что соприкосновение внешней трубы и ядра происходит по некоторой части поверхности, а не в точке про- странства. Это обстоятельство ведет к образованию застойных зон, где наблюдаются пренебрежимо малые инерционные процессы. В этом смысле определение условий для существования зазора $(a)$, где наблюдается циркуляция с учетом вязкостно-инерционно-гравитационных процессов в вязко-пластической жидкости без образования застойных зон, составляет важнейшую часть задачи исследования.

\section{Определяющие уравнения гидродинамики}

и теплообмена жидкости в кольцевом пространстве

Большинство рабочих сред (буровые жидкости, углеводородные продукты (нефти различного состава и структуры)) при транспортировке по элементам нефтегазового оборудования представляют собой реологически сложные системы, и их математическое моделирование существенно осложнено формулировкой тепло- и реофизических свойств. Закономерности 
гидродинамики и теплообмена в таких системах описываются уравнениями законов сохранения: массы (1) импульса (2) и энергии (3), физических свойств (4), которые в своей общей векторной форме, отвечающей учету рассматриваемых процессов переноса массы, импульса и энергии в вязких (ньютоновских и неньютоновских) слабосжимаемых средах, имеют вид [14-18]:

$$
\begin{gathered}
\operatorname{div} \vec{v}=0 ; \\
\frac{D \vec{v}}{D t}=\operatorname{Div} \overrightarrow{\vec{\tau}}+\vec{F} ; \\
\frac{D T}{D t}=\operatorname{div}(\operatorname{agrad} T)+S_{T} ; \\
\rho=\rho(T), c_{p}=c_{p}(T), \lambda=\lambda(T), \mu=\mu(T) .
\end{gathered}
$$

Здесь $\vec{v}$ - вектор скорости и ее компоненты: $u_{1}=u$, $u_{2}=v, u_{3}=w-$ осевая $(u)$, радиальная $(v)$ и азимутальная $(w)$ в направлении $(x, r, \varphi)$ переменных цилиндрической системы координат соответственно; $\frac{D}{D t}=\frac{\partial}{\partial t}+(\vec{v} \cdot \nabla) \equiv \frac{\partial}{\partial t}+u_{j} \frac{\partial}{\partial x_{j}}-$ полная производная; $T$ - температура транспортируемой среды; $a=\frac{\lambda}{\rho c_{p}}-$ коэффициент температуропроводности; $S_{T}$ - диссипативная функция $\left(S_{T}=\frac{1}{\rho c_{p}}\left[\vec{\tau}_{i} \cdot \frac{\partial \vec{v}}{\partial x_{i}}\right]\right) ; \overrightarrow{\vec{\tau}}-$ тензор напряжений; $\mu$ - коэффициент динамической вязкости; $\rho$ - плотность; $c_{p}-$ теплоемкость; $\vec{F}-$ массовая сила (тяжести/подъемная); $p$ - гидродинамическое давление.

\section{Модельные реологические связи} вязко-пластической жидкости

Заметим, что реологической закон изменений тензора напряжений в уравнении (2) для общего случая движения сплошных сред (включая фактор сжимаемости) можно представить в виде (5):

$$
\overrightarrow{\vec{\tau}}=-p \overrightarrow{\vec{I}}+2 \mu_{e f f}\left[\overrightarrow{\vec{S}}-\frac{1}{3}(\nabla \cdot \vec{v}) \overrightarrow{\vec{I}}\right], \quad \overrightarrow{\vec{S}}=\frac{1}{2}\left(\nabla \vec{v}+(\nabla \vec{v})^{T}\right),(5)
$$

где $\mu_{\text {eff }}$ - коэффициент эффективной вязкости следует из формулировки общего вида $-\mu_{\text {eff }}=\mu_{\text {eff }}(\overline{\bar{\tau}}, \overline{\overline{\dot{S}}}, T, P)$, причем в гетерогенных средах он также зависит от концентрации, формы и размера частиц. Учитывая, что для многих простых гомогенных (чисто сдвиговых внутренних) течений напряжение и скорость деформаций представляют собой вторые инварианты, согласно [16, 17], для характеристик вязкопластической (бингамовской) жидкости принимается (6)

$$
\bar{\tau}=\left\{\mu_{0}+\frac{\tau_{0}}{\left|[0,5(\overline{\overline{\dot{S}}} ; \overline{\overline{\dot{S}}})]^{0,5}\right|}\right\} \overline{\bar{S}} \text { при } 0,5(\bar{\tau} ; \bar{\tau})>\tau_{0}^{2}
$$

И

$$
\overline{\bar{\tau}}=\tau_{0}, \overline{\overline{\dot{S}}}=0 \text { при } 0,5(\bar{\tau} ; \bar{\tau}) \leq \tau_{0}^{2} .
$$

Анализ показывает [16-18], что при прогнозе процессов в аномально вязких гомогенных средах вполне корректным замыканием для (5) выступает, например, двухконстантная $(\kappa, m)$ модель для эффективной вязкости

$$
\mu_{e f f}=\frac{\tau_{0}(T)}{h}+\kappa(T) h^{\frac{1}{m-1}},
$$

где $\tau_{0}(T)$ - предельное напряжение сдвига в вязкопластической среде; $\kappa(T), m$ - эмпирические константы/параметры модельного реологического закона неньютоновской жидкости. Тогда как течение гетерогенных сред (смесей жидкостей с твердыми частицами) в рассматриваемых условиях следует описывать более сложными зависимостями для эффективной вязкости типа эмпирического уравнения (7), предложенного в [19]:

$$
\mu_{e f f}=\mu_{f} \exp \left\{\frac{2,5}{\beta}\left[\frac{1}{\left(1-\alpha_{s}\right)^{1,5}}-1\right]\right\}+\mu_{f r},
$$

которое для гомогенной среды редуцируется в соотношение с параметрами: $\alpha_{s}=0$ (отсутствует концентрация частиц твердой фазы); $\mu_{f}-$ коэффициент динамической вязкости транспортируемой среды в нормальных условиях; $\mu_{f r}=0$ (параметр вязкости, ответственной за трение при взаимодействии жидкой фазы с твердыми частицами); $\beta=1$ (модельный коэффициент вязкости, имеющий значение $\beta=1,5$ для гетерогенной смеси). В качестве обобщений (5) полезно отметить, что в частном случае анализа течений вязкопластической среды - бингамовской жидкости можно записать (8):

$$
\mu_{e f f} \equiv \mu_{f}=\left(\tau_{0}+\kappa \dot{\Gamma}\right) \cdot \dot{\Gamma}^{-1}, \quad \dot{\Gamma}=(0,5 \overline{\overline{\dot{S}}} \cdot \overline{\overline{\dot{S}}})^{0,5},
$$

или по [20]

$$
\mu_{\text {eff }}=\frac{\tau_{0}\left(1-\exp \left(\frac{-m \dot{\Gamma}^{n}}{\dot{S}_{0}}\right)\right.}{\dot{\Gamma}},
$$

а в случае модели Гершеля-Балкли имеем (9):

$$
\overrightarrow{\vec{\tau}}=\tau_{0}+k \overrightarrow{\dot{S}}^{n}, \quad \mu_{e f f} \equiv \mu_{f}=\left(\tau_{0}+\kappa \dot{\Gamma}^{n}\right) \cdot \dot{\Gamma}^{-1} \text {. (9) }
$$

где $\dot{S}_{0}$ - характерное значение скорости сдвига, которое в условиях подвижности стенки круглого ядра имеет вид $\dot{S}_{0}=\left[\left(\frac{W_{w}}{b}\right)^{2}+\left(\frac{U_{0}}{b}\right)^{2}\right]^{0,5} ; W_{w}=\omega R_{1}, \omega-$ угловая скорость вращения стенки ядра, $\left[\mathrm{c}^{-1}\right] ; \kappa$ - эмпирический параметр/коэффициент консистенции, [Па с $\left.{ }^{n}\right] ; n-$ индекс поведения потока, [-]; $\tau_{0}$ - предельное значение напряжения, [H/M].

\section{Формулировка краевых условий}

и детали численного решения

Получение численного решения системы определяющих уравнений с замыканиями (1)-(9) выполняется при следующих краевых условиях, отвечающих рассматриваемой конфигурации течения и теплообмена в скважине с круглым ядром, расположенным: 
1) коаксиально в условиях установившихся прямоточных и закрученных (локально на входе в кольцевую зону/методом вращающейся стенки ядра);

2) эксиентрично в условиях стационарных и неустановившихся орбитальных движений ядра (по окружности с отклонением $b$ от центра $O_{2}$ ).

\section{Начальные условия}

Так, в начальный момент времени $\left(t \in\left(t_{0}, t_{k}\right]\right.$, при $\left.t=t_{0}\right)$ в пространстве независимых переменных $(x \in[0, L]$, $\left.r \in\left(R_{1}, R_{2}\right), \varphi \in[0,2 \pi]\right)$ поля искомых параметров (компонентов вектора скорости $\left(u, v, w=F_{k}(x, r, \varphi, \mathrm{Re}, \mathrm{Ro})\right.$, $(k=x, r, \varphi)$, температуры $\left(T=T_{0}\right)$, давления $\left.\left(p=p_{0}\right)\right)$ считаются заданными с учетом характерных значений, входящих в определяющие критерии подобия: Рейнольдса $\left(\operatorname{Re}=\frac{U_{\mathrm{cp}}\left(R_{2}-R_{1}\right)}{v}\right)$, Россби $\left(\operatorname{Ro}=\frac{W_{0}}{U_{0}}\right)$, Пекле $\left(\mathrm{Pe}=\frac{U_{\mathrm{cp}}\left(R_{2}-R_{1}\right)}{a}\right)$, Фруда $\left(\mathrm{Fr}=\frac{U_{\mathrm{cp}}^{2}}{g\left(R_{2}-R_{1}\right)}\right)$, Ричардсона $\left(\mathrm{Ri}=\mathrm{Gr} / \mathrm{Re}^{2}\right)$, Грасгофа $\left(\mathrm{Gr}=\frac{g \beta_{\rho}\left(T_{w}-T_{0}\right)\left(R_{2}-R_{1}\right)^{3}}{v^{2}}\right)$, Жуковского $\left(\mathrm{Zh}=\frac{v t}{\left(R_{2}-R_{1}\right)^{2}}\right)$, Струхаля $\left(\mathrm{Sh}=\frac{U_{\mathrm{cp}} t}{\left(R_{2}-R_{1}\right)}\right)$, Фурье $\left(\right.$ Fo $\left.=\frac{a t}{\left(R_{2}-R_{1}\right)^{2}}\right)$, Прандтля $(\operatorname{Pr}=v / a)$, Бингама (для жидкости $\quad$ Гершеля-Балкли) $\quad\left(\mathrm{Bi}=\left(\frac{\tau_{0}}{\kappa}\right)\left(\frac{R_{2}-R_{1}}{U_{\text {ср }}}\right)^{n}\right)$. Причем теплофизические параметры среды представляются в виде (10):

$$
\begin{aligned}
& \frac{\rho}{\rho_{0}}=1-\beta_{\rho}\left(T-T_{w}\right) ; \frac{\mu_{0}}{\mu}=1+\beta_{\mu}\left(T-T_{w}\right) ; \\
& \frac{\lambda}{\lambda_{0}}=1-\beta_{\lambda}\left(T-T_{w}\right) ; \frac{c_{p}}{c_{p_{0}}}=1+\beta_{c}\left(T-T_{w}\right),
\end{aligned}
$$

где $\rho_{0}, c_{p 0}, \lambda_{0}-$ значения физических свойств при фиксированной температуре $T_{0} ; \beta_{\rho}, \beta_{\lambda}, \beta_{c}$ - постоянные, зависящие от рода жидкости и температурного напора. Заметим, что характерными параметрами выступают: в группе геометрически величин - продольная длина $(L) /$ ширина $(b)$ смещения центра ядра относительно продольной оси внешней трубы; в динамической части процессы со скоростью на входе $\left(U_{0}\right) /$ средней по поперечному сечению осевой составляющей скорости потока; в тепловой части - температурный напор $\left(T_{w}-T_{0}\right)$.

\section{Граничные условия}

В классе внутренних течений в кольцевой области с аксиально расположенным ядром (смещение $-b=0$; центры - $O_{2}=O_{1}$ ) предполагается, что в условиях, развивающихся по пространству и установившихся во времени прямоточных и закрученных потоков (методом вращающейся стенки ядра/локально в области входа потока в кольцевую зону с неподвижной стенкой ядра) на границах $\left(\Gamma_{i}, i=1-4\right)$, формулируются следующие граничные условия.

Ha вxоде $\left(\Gamma_{1}\right)-t \geq t_{0}, x=0, R_{1}<r<R_{2}: u=U_{0}(r) ; v=V_{0}(r)$ $w=W_{0}(r) ; T=T_{0}(r)$. Допускается, что входящий в трубу поток может быть развитым (в качестве профиля осе- вой скорости $U_{0}(r)$ выбирается профиль Пуазейля либо однородный по сечению $u=U_{0}=$ const, $v=0$, $\left.w=W_{0}(r)\right)$. Причем в условиях закрутки (локально в области входа) входящего прямоточного течения принимается: $W_{0}(r)=\omega r\left(r \in\left(R_{1}, R_{2}\right), \omega=\right.$ const $)$;

Ha выxоде $\left(\Gamma_{2}\right)-t \geq t_{0}, x=L, R_{1}<r<R_{2}: \frac{\partial \Phi_{k}}{\partial x}+\varepsilon \Phi_{k}=0$, где $\Phi_{k}=\{u, v, w, T\}$. Учитывается, что $\varepsilon \neq 0$ (некоторое малое положительное число) только для $\Phi_{\kappa}=w$.

На внешней гранище $\left(\Gamma_{3}\right)-t \geq t_{0}, x \geq 0, r=R_{2}: u=v=w=0$; $T=T_{w}$.

На стенке ядра $\left(\Gamma_{4}\right)$ формулируются условия:

- течение прямоточного потока в коаксиальном канале и локально закрученном в области входа $t \geq t_{0}, x \geq 0, r=R_{1}: u=v=w=0 ; T=T_{w} ;$

- режим прямоточного течения $(a)$, закрученного по методу вращающейся стенки ядра $(b):(a)-w=0$ при $t \geq t_{0}, x \geq 0, r=R_{1},(b)-w=W\left(R_{1}\right)=\Omega R_{1}=$ const, при $t \geq t_{0}, x \geq 0, r=R_{1}$.

- режим орбитального вращения ядра вокруг продольной оси внешней трубы.

Известно $[15,18]$, что в данных условиях течение вязкой среды (бурового раствора) по кольцевому пространству буровой колонны осложнено необходимостью учета нестационарных эффектов, вызванных смещением эксцентриситета ядра (долото с фиксированными режущими кромками), которое зависит от интенсивности вращения стенки ядра, а также специфических деформационных процессов в вязкопластической тиксотропной жидкости. Для уяснения сути этих нелинейных процессов и оценки соответствующего влияния на гидродинамику и теплообмен между стенками внешней трубы и вращающимся ядром при построении численного решения формулируется краевая задача для нестационарных неустановившихся процессов переноса массы, импульса и энергии с нелинейностью второго рода, учитывающей пространственно-временную зависимость тепло- и реофизических свойств от времени, температуры, деформационных и релаксационных процессов. Начальные условия краевой задачи, граничные условия: на входе $\left(\Gamma_{1}\right)$, на выходе $\left(\Gamma_{2}\right)$ и на внешней границе $\left(\Gamma_{3}\right)$, принимаются в виде связей, сформулированных выше. Подвижность эксцентриситета (e)/центра оси ядра $\left(O_{1}\right)$ вокруг продольной оси внешней трубы с центром $O_{2}$ за счет интенсивности вращения стенки ядра относительно своего центра $\left(O_{1}\right)$ учитывается заданием траектории центра $O_{1}(11)$ по окружности радиуса $b=O_{2}-O_{1}$ :

- при $t=t_{0}, z=z_{0}, y=y_{0}: b=b_{0}=\mathrm{const}$;

- при $t>t_{0}$ :

$$
\begin{gathered}
z(t)=b(t) \cos \left(\varphi_{\Omega}\right) ; y(t)=b(t) \sin \left(\varphi_{\Omega}\right) ; \\
\varphi_{\Omega}=\frac{\varphi t}{t_{R}^{*}} ; b(t)=\left(z^{2}(t)+y^{2}(t)\right)^{0.5} .
\end{gathered}
$$

Заметим, что в общем случае комплексного прямоточно-закрученного движения вязкой жидкости в поперечном сечении межтрубного пространства характерное время релаксационных процессов $\left(t^{*}=\frac{R_{2}-R_{1}}{U_{\text {ср }}}\right)$ соответствует следующим оценкам: 
- для течения ньютоновской среды с подвижной стенкой $-t_{R}^{*}=\frac{t^{*} \mathrm{Ro}_{w}}{W_{w}} ;$

- для неньютоновской среды при прямоточном течении $-t_{R}^{*}=\frac{\mathrm{Bi} \cdot \kappa}{\tau_{0}} ;$

- для течения неньютоновской жидкости с подвижной стенкой $-t_{R}^{*}=\left(\frac{\mathrm{Bi}}{\mathrm{Ro}_{w}}\right) \cdot\left(\frac{\kappa}{\tau_{0}}\right)$, где $W_{w}=\Omega R_{1}$, $\mathrm{Ro}_{w}=W_{w} / U_{\text {cp. }}$

Тогда, следуя идеям [21], анализ закономерностей изменений поля скорости при комплексном неустановившемся прямоточно-закрученном внутреннем течении в радиальной плоскости с эксцентричным ядром можно провести в зависимости от переменных: $r /\left(R_{2}-R_{1}\right)$ и частоты колебаний ядра в виде:

$$
\varphi t=2 M^{2} Z h, \quad M=\left(\frac{\varphi\left(R_{2}-R_{1}\right)^{2}}{2 v}\right)^{0,5},
$$

а также при $\varphi_{\text {я }}=\varphi$ Но с данными о характере гидродинамических и тепловых процессов в рассматриваемой конфигурации при критериях подобия: $\mathrm{Ho}=t / t_{R}{ }^{*}$ (совокупные нестационарные эффекты), $\mathrm{Zh}=\mathrm{Ho} / \mathrm{Re}$ (гидродинамические) и $\mathrm{Fo}=\mathrm{Zh} / \mathrm{Pr}$ (тепловые вклады). Последнее может представить интерес при обобщении результатов исследования нестационарных вязкостно-инерционно-гравитационных течений в практику приложений моделирования процессов бурения скважин, задач гидродинамики и теплообмена в нефтегазовой отрасли. Заметим, что в большинстве исследований подобных процессов моделирование проводится по следующим характерным комплексам, представляющим критерии подобия динамической части задачи о кольцевом потоке:

$$
\begin{gathered}
\Theta=\frac{R_{2}}{R_{1}}, e, n, \quad \mathrm{Bi}=\frac{\tau_{0}}{\kappa \dot{S}_{0}^{n}}, \operatorname{Re}_{s w}=\frac{\rho \omega R_{1} b}{\kappa \dot{S}_{0}^{n-1}}, \\
\operatorname{Re}=\frac{2 \rho b U_{0}}{\kappa \dot{S}_{0}^{n-1}}, U_{0}=\frac{Q}{\rho F},
\end{gathered}
$$

где $Q, F$ - соответственно расход жидкости и площадь радиального сечения кольцевой зоны. Причем диапазон изменения величин имеет границы $\Theta=0,1 \ldots 0,99, e=10-90 \%, \mathrm{Bi}=0 \ldots 2 \cdot 10^{4}$. Важным интегральным параметром течения выступает коэффициент Фаннинга (безразмерный продольный градиент давления, $\left.\frac{\partial p}{\partial x}\right)-$

$$
c_{f}=\frac{\left[2 \frac{b^{2}}{U_{0}\left(\kappa \dot{\Gamma}^{n-1}\right)} \frac{\partial p}{\partial x}\right]}{\operatorname{Re}} .
$$

\section{Особенности численного интегрирования} определяющих уравнений

Решение системы взаимосвязанных нелинейных дифференциальных уравнений в частных производных второго порядка эллиптического типа (законов сохранения массы, импульса и энергии) (1)-(4) с замыкающими соотношениями (5)-(9) выполняется численно на основе операций и блоков, используемых для: формулировки разностной сетки; дискретизации определяющих дифференциальных уравнений; получения системы линейных уравнений с параметрами в искомых разностных точках (СЛАУ); решения СЛАУ итерационным методом. Заметим, что конечноразностная дискретизация модельных уравнений выполняется с привлечением метода контрольного объема [22], который связывает дискретные аналоги определяющих уравнений с их точными интегральными балансами. Далее получение значений искомых параметров на новом слое по времени основано на использовании полностью неявной разностной схемы по времени. Как известно [23], это дает возможность уйти на шаг по времени от ограничений, которые формулируются условием устойчивости КурантаФридрихса-Леви (КФЛ), и получать решение системы уравнений на масштабах времени, определяемых процессами и условиями задачи о гидродинамике и теплообмене при транспорте реологически сложных сред во внутренних системах. Подчеркнем, что при интегрировании конвективных слагаемых уравнений законов сохранений с целью точного расчета потоков на гранях контрольного объема привлекаются противопоточные схемы: линейная первого [24] и нелинейная гибридная второго [25] порядков. Это позволяет получать корректные физические решения любых рассматриваемых в работе геометрических, гидро- и теплодинамических конфигураций, значительно минимизирует схемную вязкость и обеспечивает необходимую точность расчета параметров без их выраженных осцилляций на этапе получения установившихся решений. При аппроксимации диффузионных членов определяющих уравнений используется центрально-разносная схема. Расчет поля давления выполняется с привлечением стандартной процедуры SIMPLE algorithm [26] с сохранением положений и требований к аппроксимации уравнений [27] относительно выражений коэффициентов при сеточных узлах.

Поскольку уравнения математической модели содержат нелинейности в явном и неявном виде (через изменение рео- и теплофизических свойств от температуры, времени, релаксационных процессов в среде), линеаризация осуществляется за счет включения численной коррекции решения по методу простой итерации и организации локальных итераций для любого нелинейного уравнения. Причем глобальная итерация всегда ограничивает этот процесс при расчете поля давления и определяет переменные SIMPLE процедуры. Заметим, что в рамках рассматриваемых тепло- и гидродинамических конфигураций область пространства отличается сравнительной простотой и не требует использования сложных структурированных сеток. Суть метода конечных элементов сводится к разбиению пространства на конечное число непересекающихся контрольных объемов, включающих расчетную точку.

На рис. 1, $a-8$ представлены отдельные сведения, позволяющие судить о деталях формулировки численного метода интегрирования определяющих уравнений математической модели течения и теплообмена 
во внутренних системах. Так, расчетную область составляет определенное число контрольных объемов, и смещенные сетки используются для построения разностных аналогов для компонент вектора скорости. Такой шахматный характер распределения узлов разносной сетки для зависимых переменных позволяет исключить получение нефизичных полей. Полученные СЛАУ соответствующих дифференциальных уравнений решаются численно с применением метода расщепления/переменных направлений [26, 27], который включает на каждом шаге по пространству независимых переменных метод прогонки с трёхдиагональной матрицей. Для ускорения/замедления изменений искомых величин в итерационном процессе применяются методы последовательной верхней/нижней релаксации.
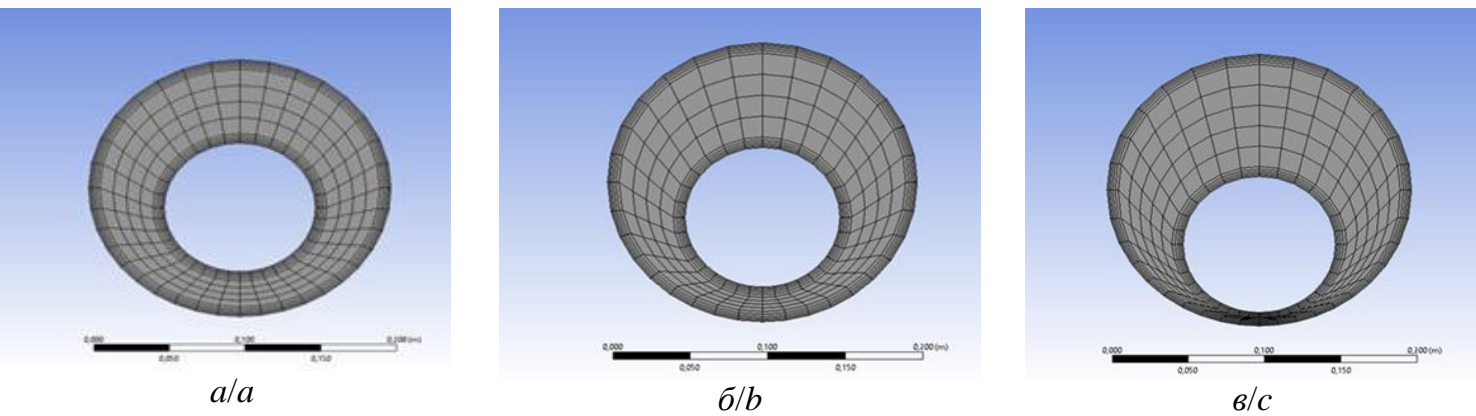

Рис. 2. Распределение узлов разностной криволинейной сетки с произвольным эксиентриситетом: а) е=30 \%; б) $e=50 \%$; в) $e=80 \%$

Fig. 2. Different grid nodes distribution with arbitrary eccentricity: a) $e=30 \%$; b) $e=50 \%$; c) $e=80 \%$

Поскольку итерационный процесс предполагает удовлетворение точным данным о явлении, для адекватной и всесторонней оценки эффективности численного решения вводится критерий точности распределения локальных и интегральных параметров процессов. Этот критерий малости между решениями, получаемыми на двух последних итерациях $(m+1, m)$ для любых искомых (тепло- и гидродинамических) параметров, отвечает виду (12):

$$
\max _{i, j, k}\left\{\frac{\left|\Phi_{i, j, k}^{m+1}-\Phi_{i, j, k}^{m}\right|}{\Phi_{i, j, k}^{m+1}}\right\} \leq \Delta_{\Phi}, \quad \Delta_{\Phi}=O\left(10^{-7}\right), \% .
$$

Кроме того, при определении точности процессов и соответствующей величины относительной погрешности $\Delta_{\phi}$ в режимах течений, для которых отсутствуют опытные данные, в качестве эталонного решения привлекается решение (для конкретной геометрической конфигурации), полученное на максимально мелкой разностной сетке. Заметим, что время сходимости возрастает с увеличением $\mathrm{Bi}$, изменением геометрии, интенсивности теплообмена. Представленные данные особенностей моделирования и деталей материальных затрат на реализацию численного алгоритма следует признать приемлемыми с точки зрения соотношения параметров иена-качествоточность в классе задач по прогнозу транспорта вязких систем и очистки скважин от шлама [28].

\section{Результаты и их обсуждение}

В работе выполнены исследования гидродинамики ньютоновской (вода) и неньютоновской жидкости (буровой раствор со свойствами жидкости типа Гершеля-Балкли, Оствальда-де-Вале) в условиях развивающихся установившихся течений на горизонтальных участках кольцевого пространства цилиндрической трубы. С целью оценок соответствия расчетных результатов имеющимся экспериментальным [29] и численным [30] данным использовались значения локальных величин процессов течения, нормированных на среднюю скорость.

Изменения определяющих геометрических, динамических и реофизических параметров задачи включают данные: $L=(100 \ldots 200) a ; R_{2}=0,05 \ldots 0,10 \mathrm{м}$; $R_{1}=0,3 \ldots 0,5$ м; плотность жидкости $\rho=850-1096 \mathrm{\kappa г} / \mathrm{m}^{3}$; коэффициент консистенции в (8) $\kappa=0,05 \ldots 0,08$ Па с ${ }^{n}$; коэффициент степенного реологического закона в (9) $n=0,5 \ldots 0,7$; предельное напряжение $\tau_{0}=5,0-8,0$ Па; $\mathrm{Re}=\frac{\rho_{f} \bar{U} b}{\mu_{f}}-$ ньютоновская реология $\left(\mathrm{Re}=0,510^{2}-10^{3}\right)$, $\operatorname{Re}=\frac{\rho_{f} \bar{U}^{2-n} D^{n}}{k}-$ неньютоновская жидкость $(\mathrm{Re}=6$ $\left.10^{2}-910^{3}\right) ; \mathrm{Bi}=\frac{\tau_{0}}{\kappa\left(\frac{\bar{U}}{D}\right)^{n}}=5-15 ; \operatorname{Pr}=4-7 ; \operatorname{Pe}=10^{1}-10^{2} ;$ $\mathrm{Fr}=10^{-4}-10^{-3} ; \mathrm{Ro}=0-3 ; \Delta T=2-15^{\circ} \mathrm{C}$.

\section{Отдельные сведения по верификации}

Задача о вязкостно-инерционном ламинарном течении ньютоновской жидкости в круглой трубе, содержащей эксцентрично расположенное круглое ядро, имеет аналитическое решение (например, [31, 32]). В сравнении со случаем автомодельного течения в трубе радиуса $R_{2}$ с коаксиально расположенным ядром $\left(R_{1}\right)$, для которого можно записать радиальное распределение осевой скорости и расхода в виде (13), (14):

$$
u=\frac{\Delta p}{4 \mu L}\left[R_{2}^{2}-r^{2}+\frac{R_{2}^{2}-R_{1}^{2}}{\ln \left(\frac{R_{2}}{R_{1}}\right)} \ln \left(\frac{r}{R_{2}}\right)\right]
$$




$$
Q=\frac{\pi \Delta p}{8 \mu L}\left[R_{2}^{4}-R_{1}^{4}-\frac{\left(R_{2}^{2}-R_{1}^{2}\right)}{\ln \left(\frac{R_{2}}{R_{1}}\right)}\right],
$$

изменение расхода $Q$ через трубу с линейными параметрами $\left(L, R_{2}, R_{1}, b\right)$ имеет вид (15), (16):

$$
Q=\frac{\pi \Delta p}{8 \mu L}\left\{\begin{array}{c}
R_{2}^{4}-R_{1}^{4}-\frac{\left[\begin{array}{l}
\left(R_{2}+R_{1}+b\right)\left(R_{2}+R_{1}-b\right) \times \\
\times\left(R_{2}-R_{1}+b\right)\left(R_{2}-R_{1}-b\right)
\end{array}\right]}{(\beta-\alpha)} \\
-4 b^{2}\left[\begin{array}{l}
R_{1}^{2}+\frac{R_{1}^{4} R_{2}^{2}}{\left(R_{2}^{2}-b^{2}\right)^{2}}+ \\
+\frac{R_{1}^{6} R_{2}^{4}}{\left\{\left(R_{2}^{2}-b^{2}\right)^{2}-R_{1}^{2} b^{2}\right\}^{2}}+\ldots
\end{array}\right]
\end{array}\right\}
$$

Здесь

$$
\begin{gathered}
\alpha=\frac{1}{2} \ln \frac{F+M}{F-M}, \quad \beta=\frac{1}{2} \ln \frac{F-b+M}{F-b-M}, \\
F=\frac{R_{2}^{2}-R_{1}^{2}+b^{2}}{2 b}, \quad M=\left(F^{2}-R_{2}^{2}\right)^{0.5} .
\end{gathered}
$$

Заметим, что в вырожденном случае (при $b=0-$ ядро размещено центрально) последний член в (13) равен нулю, и т. к. $\beta-\alpha=\ln \left(\frac{R_{2}}{R_{1}}\right)$, имеем расход при центрально расположенном ядре (17):

$$
Q_{c}=\frac{\pi \Delta p}{8 \mu L}\left[R_{2}^{4}-R_{1}^{4}-\frac{\left(R_{2}^{2}-R_{1}^{2}\right)^{2}}{\ln \left(\frac{R_{2}}{R_{1}}\right)}\right] .
$$

Учитывая соотношения (13)-(17), выполнено сравнение точности расчета структуры течения по распределению изменения расхода $\left(\frac{Q}{Q_{c}}\right)$ в зависимости от эксцентриситета $\left(e=\frac{2 b}{D-d}\right)$, где $D=2 R_{2}, d=2 R_{1}$. Причем, согласно [32], в практику прикладных исследований можно предложить критериальную связь (18):

$$
\frac{Q}{Q_{c}}=1+\frac{3 b^{2}}{2\left(R_{2}-R_{1}\right)^{2}} .
$$

Установлено, что результаты расчетов вполне успешно коррелируют с точным решением (14), (15) и связью (18), особенно при значениях $\left(R_{1} / R_{2}>0,5\right)$, а также удовлетворительно согласуются с другими известными данными, например [33-35], полученными в рамках подхода, аналогичного идеям [32], со специальным конформным отображением определяющих уравнений движения на стационарных граничных условиях, определяющих поверхности стенок внешней трубы (с радиусом $R_{2}$ ) и внутреннего ядра (с $\left.R_{1}\right)$. Заметим, что расчеты установившихся течений вязких потоков ньютоновских сред позволяют отметить, что условия для достаточно большого увеличения расхода, вызванные эксцентричностью ядра, формируются при малых зазорах (a). А также то, что малое ядро не влияет на суммарное сопротивление потоку при максимальном эксцентриситете. Также отметим, что для определения точности расчета вязкостноинерционного ламинарного течения степенной жидкости в кольцевом канале можно воспользоваться аналитическим решением [36] для осевой компоненты вектора скорости (19) в виде:

$$
u(r)=\left(\frac{\Delta p R_{2}}{2 \kappa L}\right)^{\frac{1}{n}} R_{2}\left\{\begin{array}{l}
\frac{r}{R_{2}}\left(\frac{\beta^{2}}{\alpha}-\alpha\right)^{\frac{1}{n}} d \alpha, \Theta \leq \frac{r}{R_{2}} \leq \beta ; \\
\int_{\frac{r}{R_{2}}}^{1}\left(\alpha-\frac{\beta^{2}}{\alpha}\right)^{\frac{1}{n}} d \alpha, \beta \leq \frac{r}{R_{2}} \leq 1 .
\end{array}\right.
$$

Заметим, что в (19) $\frac{\Delta p}{L}-$ перепад давления на длине кольцевой области $L$, определение параметра $\alpha$ в (19) следует из соотношения (20):

$$
\int_{\Theta}^{\beta}\left(\frac{\beta^{2}}{\alpha}-\alpha\right)^{\frac{1}{n}} d \alpha=\int_{\beta}^{1}\left(\alpha-\frac{\beta^{2}}{\alpha}\right)^{\frac{1}{n}} d \alpha .
$$

Для определения расхода предлагается критериальная связь вида (21):

$$
\begin{gathered}
Q=\frac{\pi R_{2}^{3}}{[(1 / n)+3]}\left(\frac{\Delta p}{2 \kappa L}\right)^{\frac{1}{n}} \times \\
\times\left[\left(1-\beta^{2}\right)^{\frac{1+n}{n}}-\Theta^{\frac{n-1}{n}}\left(\beta^{2}-\Theta^{2}\right)^{\frac{1+n}{n}}\right] .
\end{gathered}
$$

Расчетами установлено успешное соответствие (в условиях: $n=0,5 \ldots 0,85 ; R_{2} / R_{1}=0,5, \mathrm{Re}=70$ ) численного прогноза развития течения. Для полного анализа процессов течения аномально вязких сред можно привести аналитическое решение для ламинарного течения бингамовской жидкости в кольцевом канале [37] вида (22):

$$
u(r)=\left(\frac{\Delta p R_{2}^{2}}{2 \kappa L}\right) \times
$$

$$
\times\left\{\begin{array}{l}
-T_{0}\left(\frac{r}{R_{2}}-\Theta\right)-\frac{1}{2}\left(\left(\frac{r}{R_{2}}\right)^{2}-\Theta^{2}\right)+\beta^{2} \ln \left(\frac{r}{\Theta R_{2}}\right), \\
\Theta \leq \frac{r}{R_{2}} \leq \beta_{1} \\
-T_{0}\left(\beta_{1}-\Theta\right)-\frac{1}{2}\left(\beta_{1}^{2}-\Theta^{2}\right)+\beta^{2} \ln \left(\frac{\beta_{1}}{\Theta}\right) \\
\beta_{1} \leq \frac{r}{R_{2}} \leq \beta_{2} \\
-T_{0}\left(1-\frac{r}{R_{2}}\right)+\frac{1}{2}\left(1-\left(\frac{r}{R_{2}}\right)^{2}\right)+\beta^{2} \ln \left(\frac{r}{R_{2}}\right) \\
\beta_{2} \leq \frac{r}{R_{2}} \leq 1
\end{array}\right.
$$

Здесь обозначено:

$$
\begin{gathered}
\beta=\left(\beta_{2}^{2}-T_{0} \beta_{2}\right)^{0,5}, \quad \beta_{1}=0,5\left(\left(T_{0}^{2}+4 \beta^{2}\right)^{0,5}-T_{0}\right), \\
T_{0}=2 \tau_{0} \frac{L}{\Delta p R_{2}} .
\end{gathered}
$$


Причем для определения параметра $\beta_{2}$ привлекается уравнение (23):

$2 \beta_{2}\left(\beta_{2}-T_{0}\right) \ln \left(\frac{\beta_{2}-T_{0}}{\Theta \beta_{2}}\right)-1+\left(T_{0}+\Theta\right)^{2}+2 T_{0}\left(1-\beta_{2}\right)=0$

Расход может быть получен по соотношению (24):

$$
Q=\frac{\pi R_{2}^{4} \Delta p}{8 \kappa L}\left[\begin{array}{c}
\left(1-\Theta^{4}\right)-2 \beta_{2}\left(\beta_{2}-T_{0}\right)\left(1-\Theta^{2}\right)- \\
-\frac{4}{3}\left(1+\Theta^{3}\right) T_{0}+\frac{1}{3}\left(2 \beta_{2}-T_{0}\right)^{3} T_{0}
\end{array}\right] .
$$

Анализ данных тестирования численного алгоритма указывает, что нетривиальный характер внутренних течений в рассматриваемых условиях, осложненных переменностью реофизических свойств и наличием вращения потока, требует детального изучения особенностей изменения поля давления. В связи с этим в работе выполнялись предварительные исследования точности определения изменений перепада поля давления в канале в зависимости от средней скорости течения однородной неньютоновской жидкости в условиях экспериментов [34], где рассматривалось течение жидкости в горизонтальном полом $\left(R_{1}=0\right)$ цилиндрическом канале при параметрах: $R_{2}=0,056 \mathrm{M}, \rho=1096 \mathrm{\kappa г} / \mathrm{M}^{3}$; параметры неньютоновской жидкости - консистенция, $k_{v}=0,0747$ Па с; коэффициент степенного закона $n=0,6435$; предельное напряжение $\tau_{0}=7,34$ Па; $\quad \operatorname{Re}=\frac{\rho_{f} \bar{U}^{2-n} D^{n}}{k}=10^{3}, \quad \mathrm{Bi}=\frac{\tau_{0}}{\kappa\left(\frac{\bar{U}}{D}\right)^{n}}=8$.

Анализ показал, что имеется хорошее соответствие (погрешность меньше $10 \ldots 11$ \% при $\mathrm{U}=2,5 \ldots 3,5$ м/с) модельных результатов изменения градиента давления в зависимости от увеличения средней скорости ламинарного однородного потока при сравнении с опытными и теоретическими данными ([34]).

Важно отметить, что, поскольку предметом данного численного моделирования являются процессы переноса импульса, тепла и массы, сопровождающие движение вязких сред с продуктами выработки в режимах прямоточного и закрученного движения по кольцевой области, представляется важным получить заключения о степени влияния закрутки на локальную структуру течения. Отдельные данные расчетов течений при закрутке потоков (в области входа по закону твердого тела) и влиянии вращения на поле скорости в кольцевом пространстве трубы с центрально расположенным ядром, выполненные для режима стационарного ламинарного изотермического течения ньютоновской жидкости в отсутствии действия силы тяжести, показали следующее. Так, предлагаемая математическая модель и численный алгоритм вполне успешно предсказывает картину эволюции структуры течения из-за перестройки поля скорости вследствие затухания по длине трубы действия тангенциальной компоненты вектора скорости (подобно, например, [35]). На участке непосредственного входа в кольцевую зону $(L<1)$ получено, что действие центробежных массовых сил особенно заметно. Причем с увеличением закрутки вероятны эффекты, интенсифицирующие процессы отрыва потока от стенки ядра, и по длине трубы может формироваться локальная рециркуляционная зона. Такие особенности динамики потока ведут к изменениям интегральных параметров течения (коэффициента сопротивления трения как суммы составляющих от взаимодействия прямоточного и закрученного движений со стенкой) и повышают требования к прогнозу структуры течения у стенок (например, данные [27]).

\section{Отдельные данные расчета сложного движения в кольцевом канале \\ Ньютоновская среда}

На рис. 3-8 приведены отдельные результаты расчетов изменений динамической структуры ламинарного потока при следующих условиях: $\operatorname{Re}=10, \omega=0,009$, $1 /$ сек, $R_{2}=10 \mathrm{~cm}, R_{1}=5 \mathrm{~cm}, L=10$ м, рабочее тело - вода.

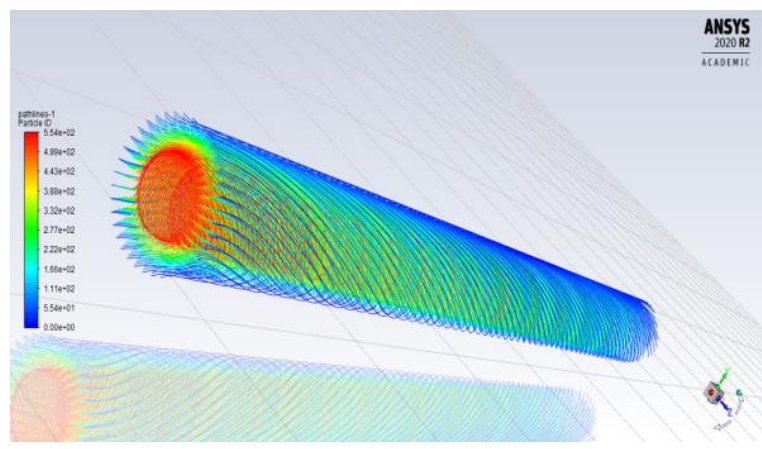

Рис. 3. Траектории линий тока при закрученном течении $(\operatorname{Re}=10)$

Fig. 3. Stream lines at the swirling flow $(R e=10)$

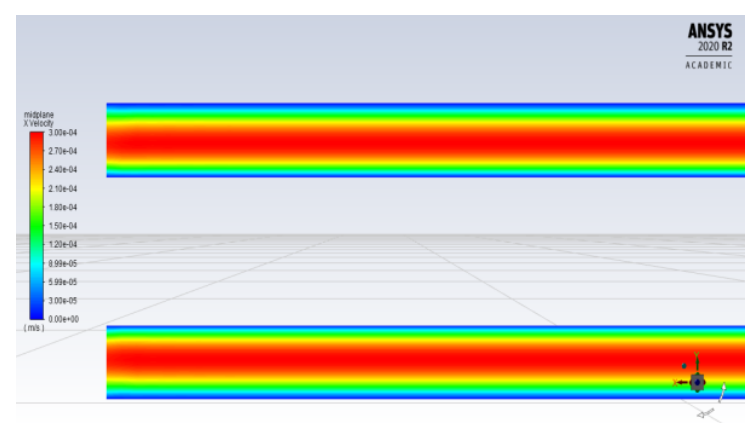

Рис. 4. Радиальное распределение осевой компоненты вектора скорости по длине кольцевого пространства с кругльм вращаюшимся ядром $\left(w=W_{w}\right)$ nри эксиентриситете $e=0$

Fig. 4. Radial distribution of axial component of velocity vector along length of annular space with round rotating core $\left(w=W_{w}\right)$ at eccentricity $e=0$

Видно, что в кольцевой области трубы с центрально расположенным ядром наложение крутки на прямоточное течение формирует специфические условия для изменений продольного градиента давления по всему поперечному сечению. Причем с увеличением значений эксцентриситета ядра изменение поля давления приводит к росту градиентов искомых величин в радиальном направлении в сравнении с их аналогами в осевом направлении. Это индуцирует рециркуляционные процессы в вязкостно-инерционном существенно неоднородном течении. 


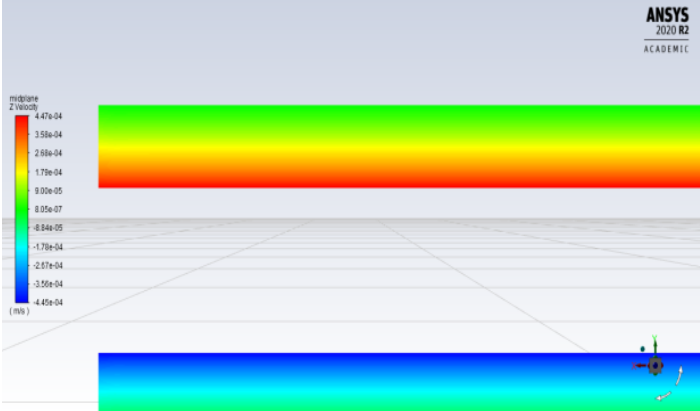

Pис. 5. Радиальное распределение тангенциильной компоненты вектора скорости по длине кольцевого пространства с кругльцм вращающчимся ядром $\left(w=W_{w}\right)$ при эксиентриситете $e=0$

Fig. 5. Radial distribution of the tangential component of the velocity vector along the length of the annular space with a round rotating core $\left(w=W_{w}\right)$ with eccentricity $e=0$

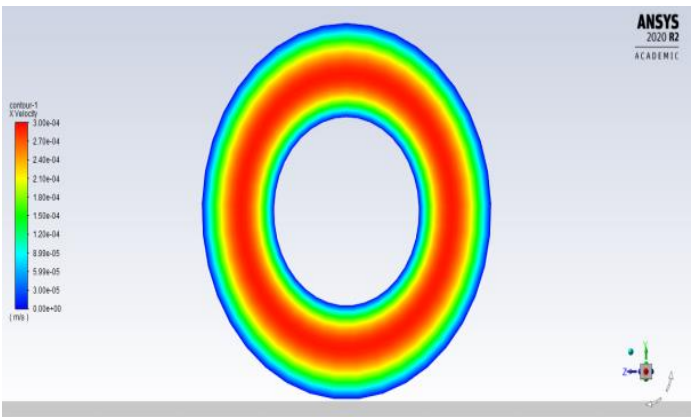

Рис. 7. Поле осевой компоненты вектора скорости в радиальном сечении кольцевой трубы при $x=1,2 \mathrm{M}, e=0$

Fig. 7. Field of axial component of velocity vector in radial section of annular pipe at $x=1,2 \mathrm{~m}, e=0$

\section{Неньютоновский поток}

Данные изменений осевого градиента давления в зависимости от эксцентриситета при прямоточном течении неньютоновской жидкости при различных значениях еe реофизических свойств в условиях: $R_{2}=0,13 \mathrm{м}, R_{1}=0,07 \mathrm{м} ;$ расход $Q=0,02 \mathrm{~m}^{3} /$ с при $U=0,85$ $\mathrm{M} / \mathrm{c}$, плотности жидкости $\rho_{f}=2750 \mathrm{\kappa} / \mathrm{m}^{3}$, параметре реологической связи $n=0,6 \ldots 1,0 ;$ коэффициент консистентности $k_{v}=0,140$ Па $\mathrm{c}^{n}, \quad \tau_{0}=2,61 \quad$ Па; $\operatorname{Re}=\frac{\rho_{f} \bar{U}^{2-n} b^{n}}{k}=10^{3} ; \quad \mathrm{Bi}=\frac{\tau_{0}}{\kappa\left(\frac{\bar{U}}{b}\right)^{n}}=1,5$, позволяют утверждать, что в условиях развитого течения увеличение эксцентриситета ядра (e) приводит к снижению градиента давления, причем более интенсивному при $n \rightarrow 1$ (ньютоновский тип жидкости, (7)). Кроме того, установлено, что подобная тенденция изменений перепада давления $\left(\frac{\partial p}{\partial x}\right)[$ Па/м] от эксцентриситета $(e)$ в неньютоновской среде сохраняется и при различных предельных значениях напряжения $\left(\tau_{0}\right)$. Это позволяет считать, что закрутка неньютоновского потока (как методом подвижной стенки ядра, так и в условиях крутки на входе) включает особенности и эффекты

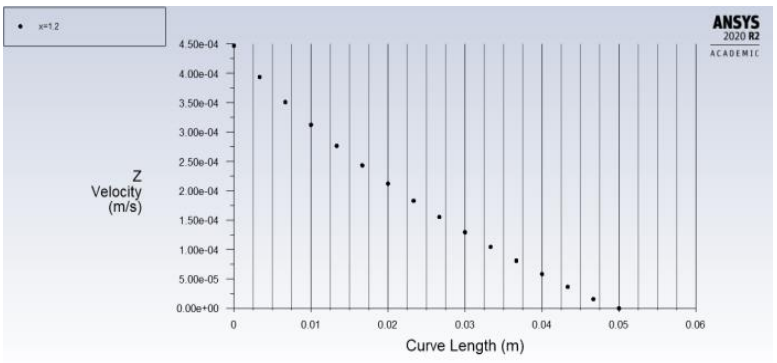

Puc. 6. Радиальное распределение тангенциальной компоненты вектора скорости в сечении по длине трубы $x=1,2 \mathrm{~N}$

Fig. 6. Radial distribution of tangential component of velocity vector in section along pipe length $x=1,2 \mathrm{~m}$

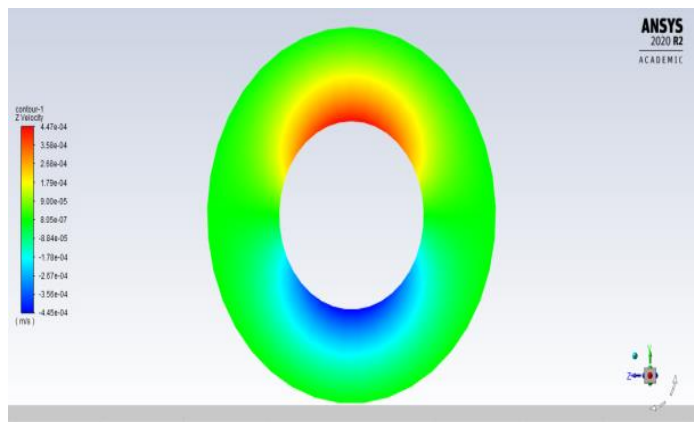

Рис. 8. Поле тангенииальной компоненты вектора скорости в радиальном сечении кольцевой трубы при $x=1,2 \mathrm{M}, e=0$

Fig. 8. Field of tangential component of velocity vector in radial section of ring pipe at $x=1,2 \mathrm{~m}, e=0$

перестройки динамической структуры вращающегося потока в прямоточное течение при определенных значениях критерия Ro. В таких условиях следует ожидать, что одновременный рост эксцентриситета и интенсивности закрутки способен генерировать условия эффективной транспортировки жидкости по кольцевому пространству. Подчеркнем, что анализируемые процессы и совместные эффекты представляют интерес для практики и требуют детального анализа, особенно для условий стационарных/нестационарных, изотермических/неизотермических течений при наличии/отсутствии действия силы тяжести. Это составляет предмет перспективных исследований при переходе к прогнозу процессов в сложных сдвиговых гомогенных и гетерогенных средах.

Режимы вращение потока, обусловленные подвижностью ядра или его стенки. Данные результатов расчета развивающегося вязкостно-инерционного ламинарного изотермического стационарного течения неньютоновской жидкости (типа Гершеля-Балкли/ Оствальда-де-Вале) указывают на существование в геометрической конфигурации ряда нетривиальных динамических эффектов, вызванных перестройкой поля скорости как в латеральной, так и радиальной областях межтрубного пространства и влиянием на 
динамику комплексов, определяющих: интенсивность вращчения (по изменению критерия Россби,

$$
\mathrm{Ro}=\frac{W_{w}}{\hat{U}},
$$

где $W_{w}=\omega R_{1}, \hat{U}$ - локальная среднемассовая скорость); взаимодействие между инериионными, вязкими силами (по критерию Рейнольдса, $\left.\mathrm{Re}=2 \rho \hat{U} a / \mu_{\text {eff }}\right) ;$ изменение фундаментальных реологических свойств эффективной вязкости $\left(\mu_{e f f}\right)$, деформаций потока (критерий Бингама,

$$
\mathrm{Bi}=\left(\frac{\tau_{0}}{\kappa}\right)\left(\frac{R_{2}-R_{1}}{U_{\mathrm{cp}}}\right)^{n},
$$

где $k, n$ - индексы постоянства и поведения потока соответственно); линейных размеров кольцевого пространства $\left(L, b, a, e, R_{1}, R_{2}\right)$.

В частности, установлено, что при малых Россби $(\mathrm{Ro}<1)$ механизмы аксиального, радиального конвективно-диффузионного переноса импульса в осевом движении превалируют в значительной части пространства. Однако в пристеночной области у поверхности ядра подвижность стенки способна генерировать эффекты, характерные для слабовязкого ламинарного течения. Последние приводят к снижению интегральных параметров течения, например, коэффициента сопротивления $\left(c_{f}\right)$, при невысоких $\mathrm{Re}$ и эксцентричности трубы $e$. Анализ режимов течений при более высоком вращении стенки (в условиях $\mathrm{Re}=\mathrm{idem}, \mathrm{Ro}=1 \ldots 5$ ) показывает, что механизмы от вращательных процессов способны формировать условия для рециркуляционных механизмов, подобно случаям закрученного вязкостного ньютоновского течения капельных жидкостей в полых круглых трубах [9, 22, 27, 35]). В таких условиях выяснение характера влияния крутки, эксцентриситета и реологических изменений в структуре жидкости на размеры и интенсивность процессов в рециркуляционных областях, выдача заключений о закономерностях течения, поведения локальных и интегральных параметров ставит вопрос о классификации рассматриваемых вязкостно-инерционных потоков, в чем нет единого мнения $[29,30,38,39]$. Расчетами обнаружено, что при высоких Ro, можно добиться уменьшения влияния низковязкого пристеночного слоя в окрестности ядра на процессы переноса в срединной части кольцевой области. Эти нелинейные эффекты между зонами низкой и высокой вязкости в пристеночной части течения у внешней стенки способны усиливать напряжения и увеличивать коэффициент сопротивления трения. Особенности этого эффекта существенно зависят от размера участка гидродинамической стабилизации, крутки стенки и потока, требуют подробного анализа с целью определения количественного значения сопротивления и установления сравнений с данными экспериментов. Уместно отметить (подобно [29, 38]), что указанный эффект достаточно выражен и при низких Re интересен анализ механизмов течения, определяющих поведение коэффициента сопротивления трения в сравнении с соответствующим данными о ньютоновском потоке. Это может составить предмет перспективных исследований.

\section{Выводы}

1. Результаты расчетов течений вязкой капельной среды, используемой для очистки горизонтальных скважин с эксцентрично расположенной бурильной трубой, указывают на нетривиальный характер зависимости ее скорости от разнообразных факторов, таких как изменение расхода, режима транспортировки без/с частицами шлама, механической скорости проходки, размеров осажденного слоя продуктов выработки, траектории скважины, интенсивности вращения бурильной трубы, а также особенностей взаимодействия смеси со стенками буровой колонны. Это требует уяснения закономерностей изменений динамической структуры аномально вязких сред, понимания физики процессов, сопровождающих перестройку прямоточно закрученного вязкостно-инерционногравитационного ламинарного течения в кольцевой области скважины.

2. Выполненное моделирование гидродинамики реологически сложного течения в межтрубном пространстве с эксцентрично расположенным круглым ядром, способным к вращению, позволяет утверждать, что на изменение структуры потока большое влияние оказывают конвективнодиффузионные и реофизические механизмы процессов переноса импульса и массы в жидкости типа Гершеля-Балкли. Эффекты, обусловленные изменением вязкости и геометрии межтрубного пространства, нелинейны и весьма чувствительны к интенсивности и специфике закрутки (методом локальной крутки потока на входе, вращением стенки круглого ядра в диапазоне изменений определяющих параметров: $\mathrm{Re}=10-10^{3}, \mathrm{Ro}=1-3$, $\mathrm{Bi}=1-15)$. Установлено, что перестройка структуры потока в прямоточное движение при течении вдоль стенки ядра может сопровождаться рециркуляционными процессами.

3. Сравнение результатов численного моделирования гидродинамики с известными экспериментальными и теоретическими данными изменений давления, расхода, кольцевой скорости (локальной и среднемассовой) от эксцентриситета, длины горизонтальных секций, условий течения показало хорошее согласие. Установлено, что в кольцевых эксцентричных областях вращение стенки круглого ядра формирует режимы, интенсифицирующие движение на горизонтальном участке скважины за счет изменений структуры и физических свойств (вязкости, плотности) буровой жидкости.

4. Расчеты показывают, что при закрутке потока (методом подвижной стенки) индуцируются циркуляционные эффекты в кольцевой области, при которых неподвижные частицы от процесса бурения в нижней части ствола скважин с эксцентричным ядром приводятся во взвешенное состояние и выносятся жидкостью из «мертвых» областей. Это явление ценно с практической точки зрения, т. к. обеспечивает контроль над функционированием дорогостоящего бурового оборудования. 
5. При внедрении результатов в практику следует учитывать, что точность моделирования и расчета процессов в рециркуляционных областях может быть улучшена посредством: включения данных об особенностях деформационных эффектов в среде и уяснения деталей течений на классе подобных геометрических конфигураций. Эти сведения представляются важными при определении оптимального режима бурильного вращения на

\section{СПИСОК ЛИТЕРАТУРЫ}

1. Hydrodynamics in unbaffled liquid-solid stirred tanks with free surface studied by DEM-VOF method / Q. Kang, D. He, N. Zhao, X. Feng, J. Wang // Journal of chemical engineering. - 2020. V. 386. - № 122846. - P. 1-17.

2. Numerical simulation of liquid droplet coalescence and breakup S. Yuan, R. Dabirian, O. Shoham, R.S. Mohan // Journal of energy resource and technology. - 2020. - V. 142. - № 10. P. 102101-1-102101-11.

3. Farakte R.A., Hendre N.V., Patwardhan A.W. CFD simulations of two phase flow in asymmetric rotary agitated columns // Industrial and engineering chemistry researches. -2018 . - V. 57. - № 50. P. 17192-17208.

4. Moghaddam A.K., Saadatabadi A.R. Rheological modeling of water based drilling fluids containing polymer/bentonite using generalized bracket formalism // Journal of petroleum science and engineering. - 2020. - V. 189. - № 107028. - P. 1-16.

5. Щукин В.К., Халатов А.А. Теплообмен, массообмен и гидродинамика закрученных потоков в полях массовых сил. - М.: Машиностроение, 1982. - 200 с.

6. Yapici S., Patrick M. A., Wragg A. A. Hydrodynamic and mass transfer in decaying annular swirl flow // International communication of heat and mass transfer. - 1994. - V. 21. P. 41-51.

7. Li H., Tomita Y. Characteristics of swirling flow in a circular pipe // Journal of fluids engineering. - 1994. - V. 116. - P. 370-373.

8. Ooms G., Kampman-Reinhartz B.E. Influence of drill pipe rotation and eccentricity on pressure drop over borehole with Newtonian liquid during drilling // SPE drill \& completion. - 2000. - V. 15. № 4. - P. 249-253.

9. Kharlamov S.N., Kim V.Yu., Silvestrov S.I. Numerical modelling of a vortical investigation of heat transfer in fields of centrifugal mass forces in elements of the power equipment with a curvilinear wall // The 5th Proceedings of the International Forum on Strategic Technology (IFOST-2010). - Ulsan, Korea, October, 13-15, 2010. - P. 105-109.

10. Heydari O., Sahraei E., Skalle P. Investigating the impact of drillpipe's rotation and eccentricity on cuttings transport phenomenon in various horizontal annuluses using computational fluid dynamics (CFD) // Journal of petroleum science and engineering. - 2017. - V. 156. - P. 801-813.

11. Steady-state cuttings transport simulation in horizontal borehole annulus / Y. Ignatenko, O. Bocharov, A. Gavrilov, R. May // ASME 2018 37th International Conference on Ocean, Offshore and Arctic Engineering. - Madrid, Spain, June 2018. - P. 26-38.

12. Формирование потока вязко-пластической жидкости в затрубном пространстве скважины / М.Ж. Ашрафян, А.И. Булатов, Г.А. Еремин, Е.А. Нелепин // Нефтяное хозяйство. 1970. - № 11. - C. 22-28.

13. Шиченко Р.И., Есьман Б.И., Кондратенко П.И. Гидравлика промывочных жидкостей. - М.: Недра, 1976. - 294 с.

14. Kharlamov S.N., Kudelin N.S., Dedeev P.O. Hydrodynamics, heat and acoustic processes modelling in transport of reologically complex viscous media technology in pipelines // XIV International Scientific Symposium in Honour of Academician M.A. Usov: PGON2014 IOP Publishing. IOP Conference Series: Earth and Environmental Science. - 2014. - V. 21. - P. 1-8. DOI: 10.1088/1755-1315/21/1/012040.

15. Харламов С.Н., Фатьянов Д.С. Исследование структуры турбулентного течения природного сырья в трубопроводах с секцией переменного по длине поперечного сечения конфузорнодиффузорного типа // Известия Томского политехнического участках горизонтальных скважин. Настоящие расчеты позволяют сформулировать методику, обеспечивающую очистку труднодоступных областей в скважине в условиях повышения скорости течения бурового раствора, поддерживаемой тангенциальными эффектами за счет включения в критериальные связи для определения скорости транспорта шлама значений Ro, Bi и эксцентриситета ядра $(e)$.

университета. Инжиниринг георесурсов. - 2020. - Т. 331. № 8. - C. 53-67.

16. Chin W.C. Computational rheology for pipeline and annular flow. - Boston: Gulf Professional Publ., 2001. - 272 p.

17. O'Donovan E.J., Tanner R.I. Numerical study of the Bingham squeeze film problem // Journal of non-Newtonian fluid mechanics. -1984 . - V. 15. - P. 75-83.

18. Процедуры и инструментарий мониторинга процессов и механизмов транспорта шлама при гидравлической очистке горизонтальных скважин / С.Н. Харламов, М.Джангхорбани // Известия Томского политехнического университета. Инжиниринг георесурсов. - 2020. - Т. 331. - № 12. - С. 22-40.

19. Cheng N.S., Law A.W.K. Exponential formula for computing effective viscosity // Powder technology. - 2003. - V. 129. P. $156-160$.

20. Papanastasiou T.C. Flow of materials with yield // Journal of rheology. - 1987. - V. 31. - P. 385-404.

21. Сигель Р., Перлмуттер М. Теплоотдача при пульсирующем ламинарном течении в канале // Теплопередача. - 1962. № 2. - P. 18-20.

22. Prospects of RANS models with multiarameter effects at simulation of complex non-isothermal flows of viscous media in devices with any configuration of surface / S.N. Kharlamov, V.Yu. Kim, S.I. Silvestrov, R.A. Alginov, S.A. Pavlov // The 6th Proc. of the International Forum on Strategic Technology (IFOST-2010). Harbin, China, August 22-24, 2011. - V. 2. - P. 787-791.

23. Anderson D.A., Tannehill J.C., Pletcher R.H. Computational fluid mechanics and heat transfer. - New York: Hemisphere Publishing Corporation, 1984. $-746 \mathrm{p}$.

24. Leonard B.P. A stable and accurate convective modelling procedure based on quadratic upstream interpolation // Computer methods in applied mechanics and engineering. - 1979. - V. 19. P. 59-98.

25. Zho J. A low-diffusive and oscillation free convection schemею // Communications in applied numerical methods. - 1991. - V. 7. P. 225-232.

26. Patankar S.V. Numerical heat transfer and fluid flow. - New York: McGraw-Hill, 1980. - $150 \mathrm{p}$

27. Бубенчиков А.М., Харламов С.Н. Математические модели неоднородной анизотропной турбулентности во внутренних системах. - Томск: Томский государственный университет, 2001. $-448 \mathrm{c}$

28. Харламов С.Н., Джангхорбани М. Процессы транспорта шлама с произвольной ориентацией буровых труб, содержащих эксцентричное расположенное круглое ядро с подвижной стенкой: проблемы, результаты, перспективы (обзор) // Известия Томского политехнического университета. Инжиниринг георесурсов. - 2020. - Т. 331. - № 7. - С. 131-149.

29. Fluid-dynamic behavior of flow in partially obstructed concentric and eccentric annuli with orbital motion / I. Bicalho, D. dos Santos, C. Ataide, C. Duarte // Journal of petroleum science and engineering. - 2016. - V. 137. - P. 202-213.

30. Gavrilov A.A., Rudyak V.Y. Reynolds-averaged modeling of turbulent flows of power-law fluids // Journal of non-Newtonian fluid mechanics. - 2016. - V. 227. - № 1. - P. 45-55.

31. Happel J., Brenner H. Low Reynolds number hydrodynamics with special applications to particular media. - New Jersey: PrentigeHall, 1965. $-553 \mathrm{p}$.

32. Piercy N.A.V., Hooper M.S., Winney H.F. Viscous flow through pipes with cores // Journal of science. - 1933. - V. 15. - P. 647-676.

33. Redberger P., Charles M. Axial laminar flow in a circular pipe containing a fixed eccentric core // Canadian Journal of chemical engineering. -1962 . - V. 40. - № 4. - P. 148-151. 
34. Kabengele K. Identification of flow patterns for coarse particles transported in a non-newtonian carrier using electrical resistance tomography: $\mathrm{PhD}$ thesis. - Capetown, SAR, 2012. $-180 \mathrm{p}$.

35. Третьяков В.В., Ягодкин В.И. Численное исследование ламинорного закрученного течения в кольцевом канале // Инженерно-физический журнал. - 1978. - Т. 34. - № 2. - С. 273-279.

36. Fredrickson A.G., Bird R.B. Non-Newtonian flow in annul // Industrial and engineering chemistry. - 1958. - V. 50. - № 3. P. 347-352.

37. Laird W.M. Slurry and suspension transport. Basic flow studies on Bingham plastic fluids // Industrial and engineering chemistry. 1957. - V. 49. - № 1. - P. 138-141.
38. Escudier M.P., Oliveira P.J., Pinho F.T. Fully developed laminar flow of purely viscous non-Newtonian liquids through annuli, including the effects of eccentricity and inner-cylinder rotation // International journal of heat and fluid flow. - 2002. - V. 23. P. 52-73.

39. Численный алгоритм для моделирования установившегося ламинарного течения неньютоновской жидкости в кольцевом зазоре с эксцентриситетом / А.А. Гаврилов, А.В. Минаков, А.А. Дектярев, В.Я. Рудяк // Вычислительные технологии. 2012. - Т. 17. - № 1. - C. 44-56.

Поступила 12.05.2021 2.

\section{Информация об авторах}

Харламов С.H., профессор, доктор физико-математических наук, профессор отделения нефтегазового дела Инженерной школы природных ресурсов Национального исследовательского Томского политехнического университета.

Джангхорбани М., аспирант отделения нефтегазового дела Инженерной школы природных ресурсов Национального исследовательского Томского политехнического университета. 
UDC 504.61:532.542: 536.252

\title{
NUMERICAL SIMULATION OF VISCOUS-INERTIAL LAMINAR SWIRLING FLOW IN A CIRCULAR TUBE WITH AN ECCENTRIC ROUND CORE
}

\author{
Sergey N. Kharlamov', \\ kharsn@mail.ru \\ Janghorbani Mehran', \\ mehran.janghorbani@gmail.com \\ 1 National Research Tomsk polytechnic university, \\ 30, Lenin avenue, Tomsk, 634050, Russia.
}

\begin{abstract}
Relevance of the research is determined by the need to understand the features of hydrodynamics and heat exchange in reologically complex homogeneous and heterogeneous media during the flow in coaxial fields. This is important for making recommendations for drilling management, improving the reliability of special equipment at high dynamic and thermal loads during the rotation of the pipe, filling the inter-tube space with sludge, as well as establishing control over changes in composition, structure, pressure, velocity and rheophysical properties (at pseudo plastic, thixotropic and viscoelastic effects) of drilling mixture on horizontal areas of wells.

Aims: to investigate the hydrodynamics of the viscous flow in coaxial tubes with eccentrically located round core, hollow channels at the conditions of direct and swirling (by the way of moving inner wall/locally at the entrance) streams; to explain the effects accompanying drilling at the sloping and horizontal sections of wells with eccentric drilling pipes; to establish the features of changes in the dynamic structure of the flow in the zones of motion of viscous media at the expense of inertial forces, as well as at the moments of flow braking when the section is cluttered by production products; to give recommendations on the beneficial effects of inertial forces on the viscosity of washing liquids to support effective oil drilling.

Methodology: the engineering analysis of the reality models of transport processes of rheologically complex viscous media in internal systems (pipes, channels) and their description by the methods of physical, mathematical and numerical modeling in the form of systems of differential and algebraic equations, the solution of which in the most important aspects of drilling is consistent with the characteristics of special equipment elements.

Results. The internal flows of viscous media with specific rheology (Newtonian and non-Newtonian fluids, such as Herschel-Bulkeley) have been investigated in geometric configurations typical of eccentric drilling pipes with effects from changes in their spatial orientation, flow rate, rotation intensity of the incoming flow/core wall, as well as rheophysical properties $(\tau, k, n)$. The parametric analysis of dynamic effects is performed for a range of criteria changes: Rossby $R o=0 \ldots 5$, Reynolds $R e=10^{2} \ldots 10^{3}$, Bingham $B i=5 \ldots 15$, eccentricity $\Delta=0,1 \ldots 0,9$. The effects of the mechanisms of convective-diffusion interaction of the momentum transfer process in pipes/channels at the complex flow movement and its contacts with walls are evaluated and generalized. Calculations show that any complications of the viscous flows are caused by changes in pressure, velocity, external and internal forces (due to the rheology). The features of the occurrence of recirculated zones at the swirling flow, fading along the length of the pipe of tangential velocity component, are analyzed. It is noted that with the growth of the core eccentricity the flow heterogeneity and the velocity vector axial component distribution asymmetry increase and the conditions to block the motion at the bottom of the inter-tube space are set. It was established that the presence of obstacles to flow movement in the coaxial fields, for example, in the form of sludge particles during drilling, can intensify the asymmetry of transfer processes, especially at high Reynolds (Re), Bingham (Bi) numbers. This can be avoided in flow regimes with the rotation of the drill pipe by the method of mobile wall/orbital movement. In conclusion, recommendations are given on modeling, calculating the flows of viscous media accompanying drilling, cleaning horizontal wells.
\end{abstract}

Key words:

Well, drilling, rotation, modeling, hydrodynamics, rheology, coaxial flows, transport, cleaning.

\section{REFERENCES}

1. Kang Q., He D., Zhao N., Feng X., Wang J. Hydrodynamics in unbaffled liquid-solid stirred tanks with free surface studied by DEM-VOF method. Journal of chemical engineering, 2020, vol. 386, no. 122846, pp. 1-17.

2. Yuan S., Dabirian R., Shoham O., Mohan R.S. Numerical simulation of liquid droplet coalescence and breakup. Journal of energy resource and technology, 2020, vol. 142, no. 10, pp. 102101-1-102101-11.

3. Farakte R.A., Hendre N.V., Patwardhan A.W. CFD simulations of two phase flow in asymmetric rotary agitated columns. Industrial and engineering chemistry researches, 2018, vol. 57, no. 50, pp. $17192-17208$

4. Moghaddam A.K., Saadatabadi A.R. Rheological modeling of water based drilling fluids containing polymer/bentonite using generalized bracket formalism. Journal of petroleum science and engineering, 2020, vol. 189, no. 107028, pp. 1-16.

5. Schukin V.K., Khalatov A.A. Teploobmen, massoobmen $i$ gidrodinamika zakruchennykh potokov $v$ polyakh massovykh sil [Heat transfer, masstransfer and hydrodynamics of swirled streams at mass forces]. Moscow, Mashinostroenie Publ., 1982. 200 p.
6. Yapici S., Patrick M.A., Wragg A.A. Hydrodynamic and mass transfer in decaying annular swirl flow. International communication of heat and mass transfer, 1994, vol. 21, pp. 41-51.

7. Li H., Tomita Y. Characteristics of swirling flow in a circular pipe. Journal of fluids engineering, 1994, vol. 116, pp. 370-373.

8. Ooms G., Kampman-Reinhartz B.E. Influence of drill pipe rotation and eccentricity on pressure drop over borehole with Newtonian liquid during drilling. SPE drill \& completion, 2000, vol. 15, no. 4, pp. 249-253.

9. Kharlamov S.N., Kim V.Yu., Silvestrov S.I. Numerical modelling of a vortical investigation of heat transfer in fields of centrifugal mass forces in elements of the power equipment with a curvilinear wall. The $5^{\text {th }}$ Proc. of the International Forum on Strategic Technology (IFOST-2010). Ulsan, Korea, October, 1-15, 2010. pp. 105-109.

10. Heydari O., Sahraei E., Skalle P. Investigating the impact of drillpipe's rotation and eccentricity on cuttings transport phenomenon in various horizontal annuluses using computational fluid dynamics (CFD). Journal of petroleum science and engineering, 2017, vol. 156, pp. 801-813. 
11. Ignatenko Y., Bocharov O., Gavrilov A., May R. Steady-state cuttings transport simulation in horizontal borehole annulus. ASME $201837^{\text {th }}$ International Conference on Ocean, Offshore and Arctic Engineering. Madrid, Spain, June 2018. pp. 26-38.

12. Aschrafyan M.J., Bulatov A.I., Eremin G.A., Nelepipn E.A. Formirovanie potoka vyazko-plastichnoy zhidkosti v zatrubnom prostranstve skvazhiny [Formation of a viscous-plastic fluid flow in the back-up space of the well]. Neftyanoe khozyaistvo, 1970, no. 11, pp. $22-28$

13. Shichenko R.I., Esman B.I., Kondratenko P.I. Gidravlika promyvochnykh zchidkostey [Hydraulics of washing liquids]. Moscow, Nedra Publ., 1976. 294 p.

14. Kharlamov S.N., Kudelin N.S., Dedeev P.O. Hydrodynamics, heat and acoustic processes modelling in transport of reologically complex viscous media technology in pipelines. XIV International Scientific Symposium in Honour of Academician M.A. Usov: PGON2014 IOP Publ. IOP Conference Series: Earth and Environmental Science, 2014, vol. 21, pp. 1-8. DOI: 10.1088/1755-1315/21/1/012040.

15. Kharlamov S.N., Fatyanov D.S. Investigation of natural raw materials turbulent flow structure in pipelines at confuser-diffuser section. Bulletin of the Tomsk Polytechnic University. Geo Assets Engineering, 2020, vol. 331, no. 8, pp. 53-67. In Rus.

16. Chin W.C. Computational rheology for pipeline and annular flow. Boston, Gulf Professional Publ., 2001. 272 p.

17. O'Donovan E.J., Tanner R.I. Numerical study of the Bingham squeeze film problem. Journal of non-Newtonian fluid mechanics, 1984, vol. 15, pp. 75-83.

18. Kharlamov S.N., Janghorbani M. Procedures and instruments for monitoring processes and mechanisms of cutting transport in hygraulic cleaning of horizontal wells. Bulletin of the Tomsk Polytechnic University. Geo Assets Engineering, 2020, vol. 331, no. 12, pp. 22-40. In Rus.

19. Cheng N.S., Law A.W.K. Exponential formula for computing effective viscosity. Powder technology, 2003, vol. 129, pp. 156160

20. Papanastasiou T.C. Flow of materials with yield. Journal of rheology, 1987, vol. 31, pp. 385-404.

21. Siegel R., Perlmutter M. Teplootdacha pri pulsiruyushchem laminarnom techenii v kanale [Heat transfer with pulsating laminar flow in the channel]. Teploperedacha, 1962, no. 2, pp. 18-32.

22. Kharlamov S.N., Kim V.Yu., Silvestrov S.I., Alginov R.A., Pavlov S.A. Prospects of RANS models with multiarameter effects at simulation of complex non-isothermal flows of viscous media in devices with any configuration of surface. The 6 ${ }^{\text {th }}$ Proc. of the International Forum on Strategic Technology (IFOST-2010). Harbin, China, August 22-24, 2011. Vol. 2, pp. 787-791.

23. Anderson D.A., Tannehill J.C., Pletcher R.H. Computational fluid mechanics and heat transfer. New York, Hemisphere Publishing Corporation, $1984.746 \mathrm{p}$

24. Leonard B.P. A stable and accurate convective modelling procedure based on quadratic upstream interpolation. Computer methods in applied mechanics and engineering, 1979, vol. 19, pp. 59-98.
25. Zho J. A low-diffusive and oscillation free convection schemeю. Communications in applied numerical methods, 1991, vol. 7, pp. 225-232.

26. Patankar S.V. Numerical heat transfer and fluid flow. New York, McGraw-Hill, 1980. $150 \mathrm{p}$.

27. Bubenchikov A.M., Kharlamov S.N. Matematicheskie modeli neodnorodnoy anizotropnoy turbulentnosti vo vnutrennikh sistemakh [Mathematical models of inhomogeneous anisotropic turbulence at the internal flows]. Tomsk, Tomsk State University Publ. House, 2001. 448 p.

28. Kharlamov S.N., Janghorbani M. Cuttings transport in hole cleaning considering well orientation, pipe eccentricity and pipe rotation: problems, results, prospects (survey). Bulletin of the Tomsk Polytechnic University. Geo Assets Engineering, 2020, vol. 331, no. 7, pp. 131-149. In Rus.

29. Bicalho I., Dos Santos D., Ataide C., Duarte C. Fluid-dymanic behavior of flow in partially obstructed concentric and eccentric annuli with orbital motion. Journal of petroleum science and engineering, 2016, vol. 137, pp. 202-213.

30. Gavrilov A.A., Rudyak V.Y. Reynolds-averaged modeling of turbulent flows of power-law fluids, Journal of non-Newtonian fluid mechanics, 2016, vol. 227, no. 1, pp. 45-55.

31. Happel J., Brenner H. Low Reynolds number hydrodynamics with special applications to particular media. New Jersey, PrentigeHall, 1965. $553 \mathrm{p}$

32. Piercy N.A.V., Hooper M.S., Winney H.F. Viscous flow through pipes with cores. Journal of science, 1933, vol. 15, pp. 647-676.

33. Redberger P., Charles M. Axial laminar flow in a circular pipe containing a fixed eccentric core. Canadian Journal of chemical engineering, 1962, vol. 40, no. 4, pp. 148-151.

34. Kabengele K. Identification of flow patterns for coarse particles transported in a non-newtonian carrier using electrical resistance tomography. PhD thesis. Capetown, SAR, 2012. $180 \mathrm{p}$.

35. Tretaykov V.V., Yagotkin V.I. Chislennoe issledovanie laminarnogo zakruchennogo techeniya $\mathrm{v}$ koltchevom kanale [Numerical investigation of laminar swirled flow in annular channel]. Inzchenerno-fizicheskiy zshurnal, 1978, vol. 34, no. 2, pp. 273-279.

36. Fredrickson A.G., Bird R.B. Non-Newtonian flow in annuli. Industrial and engineering chemistry, 1958, vol. 50, no. 3, pp. 347-352.

37. Laird W.M. Slurry and suspension transport. Basic flow studies on Bingham plastic fluids. Industrial and engineering chemistry, 1957, vol. 49, no. 1, pp. 138-141

38. Escudier M.P., Oliveira P.J., Pinho F.T. Fully developed laminar flow of purely viscous non-Newtonian liquids through annuli, including the effects of eccentricity and inner-cylinder rotation. International journal of heat and fluid flow, 2002, vol. 23, pp. 5273.

39. Gavrilov A.A., Minakov A.V., Dektyarev A.A., Rudeak V.Ya. Numerical algorithm for modeling the established laminar currents of non-Newtonian liquids in the ring gap with eccentricity. Computing technology, 2012, vol. 17, no. 1, pp. 44-56. In Rus.

Received: 12 May 2021.

\section{Information about the authors}

Sergey N. Kharlamov, Dr. Sc., professor, National Research Tomsk polytechnic university.

Janghorbani Mehran, post-graduate student National Research Tomsk polytechnic university. 